\title{
BRIEF
}

\section{An Assessment of The Interaction Between Studying Pharmacy, Problematic Use of Social Media and Depression.}

\author{
Rend Al Saigh, BSc, ${ }^{a}$ Reem Herzallah, BSc, ${ }^{a}$ Ahmed Alhusban, PharmD, PhD ${ }^{\mathrm{a}, \mathrm{b}}$ \\ ${ }^{a}$ Al Ain University, College of Pharmacy, Abu Dhabi, United Arab Emirates \\ ${ }^{\mathrm{b}}$ Jordan University of Science and Technology, Faculty of Pharmacy, Irbid, Jordan
}

Corresponding Author: Ahmed Alhusban, Jordan University of Science and Technology, Faculty of Pharmacy, Irbid, P.O. Box 3030, Irbid, Jordan 22110. Tel : +971523634829. Email : ayalhusban@just.edu.jo

Submitted March 4, 2021; accepted July 26, 2021; ePublished August 2021

Objectives. Social media applications are widely used across different age groups and communities. Problematic use of social media (PMSU) is associated with depression among university students. There is a lack of data regarding the effect of studying pharmacy on the association between PMSU and depression as compared to other university disciplines.

Methods. A cross sectional study using an online survey that recruited university students from different disciplines. PMSU was measured using Bergen Social Media Addiction Scale (BSMAS). Depression was assessed using patient health questionnaire-9 (PHQ-9).

Results. Data from 105 participants were collected with an average age of $19.88 \mathrm{SD}=2.10$ years. Forty-seven participants (44.8\%) reported depression. Pharmacy students reported lower prevalence of depression as compared to other disciplines (35.7\% and 62.9\%; respectively). Social media addiction score (OR: 1.07; 95\% CI: 1.01-1.12) and pharmacy discipline (OR: 0.2; 95\%: 0.02-0.66) were identified as independent predictors of depression. The extent of PMSU was similar between pharmacy and non-pharmacy students (49.8 and 52.7; respectively).

Conclusion. Studying pharmacy may have a differential effect on the association between PMSU and the development of depression among undergraduate university students.

Keywords: pharmacy, depression, social media addiction, education, gender.

\section{INTRODUCTION}

Mental health disorders such as depression and anxiety are established public health problem among university students ${ }^{1}$. Available data show alarmingly high prevalence of depression among university students ${ }^{1-6}$. Data from the American College Health Association survey reported that $3075(20 \%)$ of the surveyed college students had been diagnosed with depression ${ }^{1}$. Comparable numbers have been reported from other parts of the world ${ }^{7-10}$. Rotenstein et al., reported a pooled estimate of depression of about $27 \%$ among medical students ${ }^{2}$. The significance of the depression among university students is more pronounced among health care students due to the long-term consequences of depression on their college performance and future productivity ${ }^{11}$. These consequences will negatively affect the quality of care that will be provided to the general population which will impair the efficiency of the health care system.

A number of factors were suggested to explain the development of depression among university students ${ }^{7-10}$. These factors included poor academic performance, perceived stress, financial challenges, and problematic use of social media (PMSU). During the last decade, the contribution of PMSU was recognized as a significant factor in the development of depression in this group ${ }^{12-16}$. Available data consistently reported a correlation between depression and PMSU ${ }^{2,3,8,13,15,17}$. This correlation was consistent in studies that assessed students from a specific discipline ${ }^{3,12,18}$ or university students form different disciplines assessed collectively ${ }^{3,5,7-9,19}$. There is a lack of data regarding the differential effect of university discipline on the association between social media addiction and depression. Furthermore, data regarding the characteristics of PMSU and depression among pharmacy student are lacking. Accordingly, this investigation examines the association of depression and PMSU among university students with a special focus on the differential effect of studying pharmacy on this association as compared to other disciplines.

\section{METHODS}

This study is a cross sectional study to assess the association between PMSU and depression among university students. A special focus of the study is placed on assessing the differential effect of studying pharmacy on the association between PMSU and depression. 
Participants were recruited through an open online invitation that was circulated using the mailing lists and social media groups across different colleges in Al Ain University, Abu Dhabi, UAE. Al Ain University is a private university with six colleges including Engineering, Pharmacy, Education, Media, Law and Business. All potential participants were offered a description of the study and their ability to leave the study at any time was stressed. The study protocol was approved by the respective authority in the university.

Participant's demographic data including age, gender, discipline, and university level were collected from each participant. The university level was categorized into Junior for first year students and advanced for other years. This categorization was based on published literature ${ }^{7,12,15,17,20}$. The assessment of PMSU was conducted using Bergen Social Media Addiction Scale (BSMAS), a modified version of the Arabic version of Bergen Facebook addiction scale (BFAS) after getting the permission of the author ${ }^{21}$. The Arabic version was modified by replacing the word Facebook with social media as suggested and validated by Andreessen et al ${ }^{14}$. The tool is composed of 18 items measured on a 5-point Likert scale ranging from never to very often. The scale is calculated by summing the score of each item that are assigned a value of 1-5. The scale ranges from 18-90 with higher values indicate higher levels of PMSU.

Depression symptoms were assessed using the Arabic version of patient health questionnaire-9 (PHQ-9). The PHQ-9 is self-reported version of the PRIME-MD diagnostic tool ${ }^{22}$. It has of 9 items measured on a 4-points Likert scale ranging from "not at all: to "nearly every day". The scale score ranges from 0 to 27 and a cutoff point of more than 9 has been suggested to identify students with depression.

\section{Statistical analysis}

Descriptive statistics were calculated for the demographic variables. The distribution of the depression across the different demographic data variables was calculated using the $\chi 2$ for categorial variables. Student's t-test or one-way analysis of variance (ANOVA) applying Bonferroni as a post hoc analysis as appropriate were used for continuous variables. Odds ratio and the corresponding 95\% confidence intervals were calculated for the presence of depressive symptoms using a stepwise binary logistic regression analysis model. A liner regression model was used for the extent PMSU using a forward least regression approach. All statistical analyses were performed using the statistical package for social sciences (SPSS) version 24 (IBM SPSS Statistics for Windows, Version 24.0. Armonk, NY: IBM Corp.). A p value of less than 0.05 was considered statistically significant.

\section{RESULTS \\ Patient Demographics}

A total of 105 participants were recruited in this study with an average age of $19.88 \mathrm{SD}=2.10$ years. The majority of participants were females (73.3\%). The study included $70(66.7 \%)$ pharmacy students and 72 of participants were advanced (68.6\%). The average BSMAS score of the sample was $50.76(\mathrm{SD}=11.8)$. Table 1 provides detailed description of the basic demographics of the participants.

\section{The Prevalence of Depression Symptoms}

In this study, 47 (44.76\%) participants reported PHQ-9 scores consistent with depression (a score of 9 or higher in the PHQ-9). The average age of the participants with depression was similar to participants without depression (19.75(SD=1.4) vs. 19.98( $\mathrm{SD}=2.5) ; p=.58)$. Similarly, the gender distribution between both groups was similar $(p=.49)$. Pharmacy students comprised a significantly smaller proportion of the subjects with depression as compared to nonpharmacy students (35.7 vs. 64.3; $p=.008$ ). Furthermore, participants with depression scored significantly higher on the BSMAS scale (55.4 ( $\mathrm{SD}=10.36)$ vs. $47(\mathrm{SD}=11.67) ; p=.001)$. Table 1 provides detailed description of the characteristics of participants and according to their reported mood state.

\section{Predictors of Reporting Depression Symptoms}

A binary logistic regression analysis that corrected for age, gender, discipline, university level, the used social media applications and BSMAS, was used to identify the independent predictors of reporting depression. In this analysis the university discipline and the BSMAS score were identified as independent predictors of depression.

Pharmacy students were less likely to report have depression (OR: 0.203, 0.062-0.664; $p=.008$ ). Furthermore, higher BSMAS score increased the likelihood of reporting depression (OR: 1.07, 1.021-1.122; $p=.005$ ). Table 2 provides a detailed description of the analysis.

\section{Predictors of Social Media Applications Addiction}

To determine the predictors of social media addiction a stepwise linear regression model that corrected for age, gender, discipline, university level and PHQ-9 score. The final model was able to analyze $33.6 \%$ of the variability in the 
model $(\mathrm{F}=9.7 ; p=<.001)$ PHQ-9 score was identified as the sole predictor of PMSU (Standardized Coefficients Beta= $0.512,0.687-1.395 ; p<.001)$. Table 2 provides a detailed description of the model.

\section{The Characteristics Of The Participants According To The Discipline}

To further the understanding of the effect of discipline on reporting the symptoms of depression, a comparison of the participant's characteristics based on the discipline was made (Table 4). Pharmacy students had a similar average age to participants from other disciplines (20.12 ( $\mathrm{SD}=2.29)$ vs. 19.41( $\mathrm{SD}=1.59) ; p=.11)$. Similarly, both groups reported comparable results on the social media addiction scale (49.77 ( $\mathrm{SD}=12.54)$ vs. $52.74(\mathrm{SD}=10.13) ; p=.23)$.

Females comprised a larger proportion of pharmacy students as compared to other disciplines (85.7 vs. 48.6; $p<.001)$. Furthermore, a majority of pharmacy students were advanced (77.1 vs. $51.4 ; p=.007)$.

\section{DISCUSSION}

In this manuscript, we are reporting on the association between PMSU and the prevalence of depression among university students with a special focus on pharmacy students. Our data suggests high prevalence of depressive symptoms among university students. The level of PMSU and the discipline were identified as independent predictors of depression. Pharmacy students were less likely to be depressed and students with high level of PMSU are more likely to report symptoms of depression.

The prevalence of depression has been identified as an alarming health issue worldwide ${ }^{1-3,5,10,23}$. Available data reported different prevalence rates of depression among university students that ranged from $27 \%$ to $51 \%{ }^{27,8,10,24}$. These estimates are consistent with our estimates of about $45 \%$ of the sample. Furthermore, we identified the extent of PMSU and educational discipline as independent predictors of reporting depression.

In this study, special attention was placed on assessing the differential effect of studying pharmacy on the association between PMSU and depression. The prevalence of depression in our study was $35.7 \%$ which is consistent with the range of previously reported rates among pharmacy students. Abbas et al., reported a $62 \%$ prevalence of depression in Pakistani pharmacy students whereas Silva et al., reported a lower prevalence of depressive symptoms that was less than the cutoff point for depression among Portuguese pharmacy students ${ }^{25,26}$. Similar studies in the United States reported a $19.3 \%-29.5 \%{ }^{27,28}$. This variability was attributed to country specific socioeconomic factors. Furthermore, our data demonstrated a lower rate of depression among pharmacy students as compared to non-pharmacy students. In contrast, Fischbein et al., reported a similar prevalence of depression between pharmacy and medical students ${ }^{28}$. Similarly, Sabourin et al. reported similar prevalence of depression between pharmacy and non-pharmacy university students ${ }^{27}$. This discrepancy might be attributed to differences in the prevalence of depression in the three studies. In the aforementioned studies, the prevalence of depression was less than $20 \%$ whereas our data reported a prevalence of more than $45 \%$ in the overall sample. This variability might be influenced by country specific factors and the small sample size of our study. Additionally, a significantly higher proportion of the pharmacy students in our study were seniors and females as compared to non-pharmacy students. The larger number of senior students may explain the lower prevalence of depression among pharmacy students as it has been reported that freshman students faced more issues with adaptation and were more likely to report depressive symptoms ${ }^{12,15}$. Counterintuitively, females, who represented the majority of recruited pharmacy students have been consistently reported to be more likely to report depression ${ }^{13,14,29,30}$. This discrepancy requires further investigation. Furthermore, self-esteem and academic stress were linked to the development of depression among university students. There is a need to investigate the contribution of these factors to the lower prevalence of depression in our sample.

In a large study, Andreassen et al., reported a positive correlation between the extent of social media use and the prevalence of depression ${ }^{14}$. Similarly, there is a plethora of evidence supporting the association between PMSU and the development of depression among young adults, particularly university students ${ }^{12,13,18,29,31,32}$. Available literature suggested female gender, depression, and anxiety as predictors of PMSU ${ }^{12-14,29}$. Our findings did not show an increased risk of PMSU among females. On the other hand, the presence of depression was the only independent predictor of social media addiction. This finding is consistent with reported data regarding the predictors of PMSU ${ }^{12,15,17,29,30}$. Furthermore, the extent of PMSU was similar between pharmacy and non-pharmacy students in our sample. This finding is consistent with identifying depression, which was lower in pharmacy students, as the only independent predictor of problematic use of social media applications.

The number of social media applications and their features is large and continuously increasing. Accordingly, it's prudent to assess the effect of the type of application used on depression and problematic social media use. The prevalence of depression and the extent of problematic social media use were not altered by the type of social media application used. Data regarding the effect of social media application is limited except for a study that suggested an 
increased risk of depression and social media addiction among individuals who use twitter more than other applications. More research is needed in this specific area.

\section{Limitation}

We acknowledge a number of limitations in this work. The study was performed in a single educational institution with a small number of participants which might limit the generalizability of the results. Additionally, the use of selfreported questionnaire may introduce bias.

\section{CONCLUSION}

In conclusion, social media addiction is a predictor of depression among university students and pharmacy students are less likely to have depression $(35.7 \%$ Vs. $62.9 \%)$. The contribution of university level and gender to the development of depression maybe altered by the discipline.

\section{ACKNOWLEDGMENTS}

The author would like to thank Dr. Laila Alhusban for the assistance in language proofing and editing of the study.

\section{REFERENCES}

1. American College Health Association. American College Health Association-National College Health Assessment III: Reference Group Executive Summary Fall 2020.; 2020.

2. Rotenstein LS, Ramos MA, Torre M, et al. Prevalence of Depression, Depressive Symptoms, and Suicidal Ideation Among Medical Students. JAMA. 2016;316(21). doi:10.1001/jama.2016.17324

3. Lun KW, Chan C, Ip PK, et al. Depression and anxiety among university students in Hong Kong. Hong Kong Medical Journal. Published online September 24, 2018. doi:10.12809/hkmj176915

4. January J, Madhombiro M, Chipamaunga S, Ray S, Chingono A, Abas M. Prevalence of depression and anxiety among undergraduate university students in low- and middle-income countries: a systematic review protocol. Systematic Reviews. 2018;7(1). doi:10.1186/s13643-018-0723-8

5. Ibrahim AK, Kelly SJ, Adams CE, Glazebrook C. A systematic review of studies of depression prevalence in university students. Journal of Psychiatric Research. 2013;47(3). doi:10.1016/j.jpsychires.2012.11.015

6. Rith-Najarian LR, Boustani MM, Chorpita BF. A systematic review of prevention programs targeting depression, anxiety, and stress in university students. Journal of Affective Disorders. 2019;257. doi:10.1016/j.jad.2019.06.035

7. Koly KN, Sultana S, Iqbal A, Dunn JA, Ryan G, Chowdhury AB. Prevalence of depression and its correlates among public university students in Bangladesh. Journal of Affective Disorders. 2021;282. doi:10.1016/j.jad.2020.12.137

8. Shamsuddin K, Fadzil F, Ismail WSW, et al. Correlates of depression, anxiety and stress among Malaysian university students. Asian Journal of Psychiatry. 2013;6(4). doi:10.1016/j.ajp.2013.01.014

9. Eisenberg D, Gollust SE, Golberstein E, Hefner JL. Prevalence and correlates of depression, anxiety, and suicidality among university students. American Journal of Orthopsychiatry. 2007;77(4). doi:10.1037/00029432.77.4.534

10. Akhtar P, Ma L, Waqas A, et al. Prevalence of depression among university students in low and middle income countries (LMICs): a systematic review and meta-analysis. Journal of Affective Disorders. 2020;274. doi:10.1016/j.jad.2020.03.183

11. Cain J. It's Time to Confront Student Mental Health Issues Associated with Smartphones and Social Media. American Journal of Pharmaceutical Education. 2018;82(7). doi:10.5688/ajpe6862

12. Yücens B, Üzer A. The relationship between internet addiction, social anxiety, impulsivity, self-esteem, and depression in a sample of Turkish undergraduate medical students. Psychiatry Research. 2018;267. doi:10.1016/j.psychres.2018.06.033

13. Shensa A, Escobar-Viera CG, Sidani JE, Bowman ND, Marshal MP, Primack BA. Problematic social media use and depressive symptoms among U.S. young adults: A nationally-representative study. Social Science \& Medicine. 2017;182. doi:10.1016/j.socscimed.2017.03.061

14. Andreassen CS, Billieux J, Griffiths MD, et al. The relationship between addictive use of social media and video games and symptoms of psychiatric disorders: A large-scale cross-sectional study. Psychology of Addictive Behaviors. 2016;30(2). doi:10.1037/adb0000160 
15. Zhou M, Li F, Wang Y, Chen S, Wang K. Compensatory Social Networking Site Use, Family Support, and Depression Among College Freshman: Three-Wave Panel Study. Journal of Medical Internet Research. 2020;22(9). doi:10.2196/18458

16. Ghaemi SN. Digital depression: a new disease of the millennium? Acta Psychiatrica Scandinavica. 2020;141(4). doi:10.1111/acps.13151

17. Tang CS, Koh YYW. Online social networking addiction among college students in Singapore: Comorbidity with behavioral addiction and affective disorder. Asian Journal of Psychiatry. 2017;25. doi:10.1016/j.ajp.2016.10.027

18. Naja WJ, Kansoun AH, Haddad RS. Prevalence of Depression in Medical Students at the Lebanese University and Exploring its Correlation With Facebook Relevance: A Questionnaire Study. JMIR Research Protocols. 2016;5(2). doi:10.2196/resprot.4551

19. Regehr C, Glancy D, Pitts A. Interventions to reduce stress in university students: A review and meta-analysis. Journal of Affective Disorders. 2013;148(1). doi:10.1016/j.jad.2012.11.026

20. Ibrahim AK, Kelly SJ, Adams CE, Glazebrook C. A systematic review of studies of depression prevalence in university students. Journal of Psychiatric Research. 2013;47(3). doi:10.1016/j.jpsychires.2012.11.015

21. Salem AAMS, Almenaye NS, Andreassen CS. A Psychometric Evaluation of Bergen Facebook Addiction Scale (BFAS) of University Students. International Journal of Psychology and Behavioral Sciences. 2016;6(5):199-205.

22. Kroenke K, Spitzer R, Williams J. The PHQ - 9: validity of a brief depression severity measure. J Gen Intern Med. 2001;16(9):606-613.

23. Lazarevich I. Depression and food consumption in Mexican college students. Nutrición Hospitalaria. Published online May 10, 2018. doi:10.20960/nh.1500

24. Mamun MA al, Griffiths MD. The association between Facebook addiction and depression: A pilot survey study among Bangladeshi students. Psychiatry Research. 2019;271. doi:10.1016/j.psychres.2018.12.039

25. Silva RG, Figueiredo-Braga M. Evaluation of the relationships among happiness, stress, anxiety, and depression in pharmacy students. Currents in Pharmacy Teaching and Learning. 2018;10(7). doi:10.1016/j.cptl.2018.04.002

26. Abbas A, Rizvi SA, Hassan R, et al. The prevalence of depression and its perceptions among undergraduate pharmacy students. Pharmacy Education. 2015;15(1).

27. Sabourin AA, Prater JC, Mason NA. Assessment of mental health in doctor of pharmacy students. Currents in Pharmacy Teaching and Learning. 2019;11(3). doi:10.1016/j.cptl.2018.12.004

28. Fischbein R, Bonfine N. Pharmacy and Medical Students' Mental Health Symptoms, Experiences, Attitudes and Help-Seeking Behaviors. American Journal of Pharmaceutical Education. 2019;83(10). doi:10.5688/ajpe7558

29. Nesi J, Prinstein MJ. Using Social Media for Social Comparison and Feedback-Seeking: Gender and Popularity Moderate Associations with Depressive Symptoms. Journal of Abnormal Child Psychology. 2015;43(8). doi:10.1007/s10802-015-0020-0

30. Wong HY, Mo HY, Potenza MN, et al. Relationships between Severity of Internet Gaming Disorder, Severity of Problematic Social Media Use, Sleep Quality and Psychological Distress. International Journal of Environmental Research and Public Health. 2020;17(6). doi:10.3390/ijerph17061879

31. Bhandari PM, Neupane D, Rijal S, Thapa K, Mishra SR, Poudyal AK. Sleep quality, internet addiction and depressive symptoms among undergraduate students in Nepal. BMC Psychiatry. 2017;17(1). doi:10.1186/s12888017-1275-5

32. Jeri-Yabar A, Sanchez-Carbonel A, Tito K, et al. Association between social media use (Twitter, Instagram, Facebook) and depressive symptoms: Are Twitter users at higher risk? International Journal of Social Psychiatry. 2019;65(1). doi:10.1177/0020764018814270 
Table 1. Baseline Characteristics of the Participants

\begin{tabular}{|c|c|c|c|c|}
\hline & Total & Depression $(\mathrm{N}=47)$ & $\begin{array}{l}\text { Normal Mood } \\
\qquad(\mathrm{N}=58)\end{array}$ & $p$-value \\
\hline Age(years), mean \pm SD & $19.9 \pm 2.1$ & $19.8 \pm 1.4$ & $19.98 \pm 2.5$ & .58 \\
\hline \multicolumn{5}{|l|}{ Gender, n (\%) } \\
\hline Male & $28(26.7)$ & $11(39.3)$ & $17(60.7)$ & \multirow[t]{2}{*}{.49} \\
\hline Female & $77(73.3)$ & $36(46.8)$ & $41(53.2)$ & \\
\hline \multicolumn{5}{|l|}{ Discipline, n (\%) } \\
\hline Pharmacy & $70(66.7)$ & $25(35.7)$ & $45(64.3)$ & \multirow[t]{2}{*}{.008} \\
\hline Other & $35(33.3)$ & $22(62.9)$ & $13(37.1)$ & \\
\hline \multicolumn{5}{|l|}{ University Level ${ }^{\mathrm{a}}, \mathrm{n}(\%)$} \\
\hline Junior & $33(31.4)$ & $15(45.5)$ & $18(54.5)$ & \multirow[t]{2}{*}{.92} \\
\hline Advanced & $72(68.6)$ & $32(44.4)$ & $40(55.6)$ & \\
\hline \multicolumn{5}{|l|}{ Social Media Apps, n (\%) } \\
\hline Instagram & $62(59)$ & $26(41.9)$ & $36(58.1)$ & \multirow[t]{5}{*}{.92} \\
\hline Snapchat & $10(9.5)$ & $4(40)$ & $6(60)$ & \\
\hline WhatsApp & $20(19)$ & $10(50)$ & $10(50)$ & \\
\hline Twitter & $11(10.5)$ & $6(54.5)$ & $5(45.5)$ & \\
\hline Facebook & $2(1.9)$ & 1 & 1 & \\
\hline $\mathrm{BSMAS}^{\mathrm{b}}$, mean $\pm \mathrm{SD}$ & $50.8 \pm 11.8$ & $55.4 \pm 10.4$ & $47 \pm 11.7$ & .001 \\
\hline
\end{tabular}


Table 2. Predictors of Depression and Problematic Use of Social Media Among University Students

\begin{tabular}{|c|c|c|c|c|c|c|}
\hline & \multicolumn{2}{|c|}{ Depression } & \multicolumn{3}{|c|}{ Problematic Use of Social Media ${ }^{\mathrm{g}}$} & \multirow[b]{2}{*}{ p-value } \\
\hline & $\mathbf{O R}^{\mathrm{e}}$ & $95 \% \mathrm{CI}^{\mathrm{f}}$ & p-value & $\begin{array}{c}\text { Standardized } \\
\text { Coefficients Beta }\end{array}$ & $95 \% \mathrm{CI}$ & \\
\hline Age (years) & 0.9 & $0.7-1.2$ & 0.4 & 0.04 & $-0.9-1.2$ & 0.7 \\
\hline Gender $^{\mathrm{a}}$ & 2.6 & $0.7-9.3$ & 0.1 & 0.2 & $-1.1-8.8$ & 0.1 \\
\hline Discipline $^{\mathrm{b}}$ & 0.2 & $0.1-0.7$ & 0.008 & 0.1 & $-1.5-7.7$ & 0.2 \\
\hline University Level $^{\mathrm{c}}$ & 1.7 & $0.5-5.5$ & 0.4 & -0.1 & $-7.1-2.9$ & 0.4 \\
\hline Social Media Apps ${ }^{\mathrm{d}}$ & 1.3 & $0.91-1.9$ & 0.2 & - & - & - \\
\hline BSMAS & 1.1 & $1.0-1.1$ & 0.005 & - & - & - \\
\hline Depression Score & - & - & - & 0.5 & $0.7-1.4$ & $<0.001$ \\
\hline
\end{tabular}

${ }^{\mathrm{a}}$ The reference group is males.

${ }^{b}$ The reference group is non-pharmacy.

${ }^{c}$ The reference group is Junior.

${ }^{\mathrm{d}}$ The reference group is Instagram.

${ }^{\mathrm{e}}$ Odds ratio.

${ }^{\mathrm{f}}$ Confidence interval.

${ }^{\mathrm{g}}$ Adjusted $\mathrm{R}^{2}=0.3 ; \mathrm{F}=9.7, p=<.001$

BSMAS: Bergen Social Media Addiction Scale 
Table 3. Characteristics of Participants Stratified According to University Discipline

\begin{tabular}{|c|c|c|c|}
\hline & Pharmacy $(\mathrm{N}=70)$ & Other $(\mathrm{N}=35)$ & p-value \\
\hline Age(years), mean \pm SD & $20.1 \pm 2.3$ & $19.4 \pm 1.6$ & 0.1 \\
\hline \multicolumn{4}{|l|}{ Gender, n (\%) } \\
\hline Male & $10(14.3)$ & $18(51.4)$ & \multirow[t]{2}{*}{$<0.001$} \\
\hline Female & $60(85.7)$ & 17 (48.6) & \\
\hline \multicolumn{4}{|l|}{ University Level ${ }^{\mathrm{a}}, \mathrm{n}(\%)$} \\
\hline Junior & $16(22.9)$ & $17(48.6)$ & \multirow[t]{2}{*}{0.007} \\
\hline Advanced & $54(77.1)$ & $18(51.4)$ & \\
\hline \multicolumn{4}{|l|}{ Social Media Apps, n (\%) } \\
\hline Instagram & $42(60)$ & $20(57.1)$ & \multirow[t]{5}{*}{0.3} \\
\hline Snapchat & $4(5.7)$ & $6(17.1)$ & \\
\hline WhatsApp & $16(22.9)$ & $4(11.4)$ & \\
\hline Twitter & $7(10)$ & $4(11.4)$ & \\
\hline Facebook & $1(1.4)$ & $1(2.9)$ & \\
\hline $\mathrm{BSMAS}^{\mathrm{b}}$, mean $\pm \mathrm{SD}$ & $49.8 \pm 12.5$ & $52.7 \pm 10.1$ & 0.2 \\
\hline Depression $^{\mathrm{c}}, \mathrm{n}(\%)$ & $25(35.7)$ & $22(62.9)$ & 0.008 \\
\hline
\end{tabular}

${ }^{\mathrm{a}}$ The university level is categorized into junior and advanced which includes students from the study years beyond first year.

${ }^{\mathrm{b}}$ BSMAS: Bergen Social Media Addiction Scale: is used to quantify the extent of problematic use of social media. Higher scores reflect higher extent of problematic use.

${ }^{\mathrm{c}}$ Depression is assessed using the PHQ-9 scale where participants scoring 9 or higher are identified as having depression. 